\title{
INTERNATIONAL JOURNAL OF HUMANOID ROBOTICS
}

\section{Author Index Volume 5 (2008)}

Acosta-Calderon, C. A., Mohan, R. E., Zhou, C., Hu, L., Yue, P. K. and Hu, H., A Modular Architecture for Humanoid Soccer Robots with Distributed Behavior Control

Armstrong-Crews, N., see Veloso, M.

Asada, M., see Mayer, N. M.

Asfour, T., Azad, P., Gyarfas, F. and Dillmann, R., Imitation Learning of Dual-Arm Manipulation Tasks in Humanoid Robots

Azad, P., see Asfour, T.

Başeski, E., see Kraft, D.

Bauer, A., Wollherr, D. and Buss, M., Human-Robot Collaboration: A Survey

Bebis, G., see Kelley, R.

Behnke, S. and Stückler, J., Hierarchical Reactive Control for Humanoid Soccer Robots

Billard, A., see Hersch, M.

Borovac, B., see Vukobratović, M.

Borovac, B., see Vukobratović, M.

Burke, J., see Nomura, T.

Buss, M., see Bauer, A.

Carpin, S., Kallmann, M. and Pagello, E., The Challenge of Motion Planning for Soccer Playing Humanoid Robots

Chella, A., see Menegatti, E.

Cheng, G., see Morén, J.

Cheng, G., see Ude, A.

Chernova, S., see Veloso, M.

Cisternino, A., see Menegatti, E.

Crawford, E., see Veloso, M.

Davison, A., see Stasse, O.

Dillmann, R., see Asfour, T.

Erdemir, E., see Kawamura, K.

Esteves, C., see Stasse, O.

Fan, X. and Henderson, T. C., RobotShare: A Google for Robots

Friedmann, M., Kiener, J., Petters, S., Thomas, D., Stryk, O. V. and

Sakamoto, H., Versatile, High-Quality Motions and Behavior Control of a Humanoid Soccer Robot

Gordon, S. M., see Kawamura, K.

Greggio, N., see Menegatti, E.

Gyarfas, F., see Asfour, T.

Hagita, N., see Hayashi, K.

Hagita, N., see Shiomi, M.

Hall, J. F., see Kawamura, K.

Han, J., see Nomura, T.

$\mathbf{3}$ (2008) 397

3 (2008) 457

3 (2008) 335

2 (2008) 183

2 (2008) 183

2 (2008) 247

1 (2008) 47

2 (2008) 203

3 (2008) 375

2 (2008) 161

1 (2008) 119

4 (2008) 639

1 (2008) 25

1 (2008) 47

3 (2008) 481

3 (2008) 523

1 (2008) 3

2 (2008) 267

3 (2008) 457

3 (2008) 523

3 (2008) 457

2 (2008) 287

2 (2008) 183

4 (2008) 547

2 (2008) 287

2 (2008) 311

3 (2008) 417

$4(2008) 547$

3 (2008) 523

$2(2008) 183$

1 (2008) 67

$4(2008) 587$

$4(2008) 547$

1 (2008) 25 
Hayashi, K., Kanda, T., Miyashita, T., Ishiguro, H. and Hagita, N., Robot Manzai: Robot Conversation as a Passive-Social Medium

$1(2008) 67$

$2(2008) 311$

$4(2008) 639$

Herr, H. M., see Vukobratović, M.

Hersch, M., Sauser, E. and Billard, A., Online Learning of the Body Schema

Hofmann, A., see Vukobratović, M.

$\mathrm{Hu}, \mathrm{H}$., see Acosta-Calderon, C. A.

$\mathrm{Hu}$, L. and Zhou, C., Gait Generation and Optimization Using the Estimation of Distribution Algorithm for TeenSize Humanoid Soccer Robot RESr-1

$\mathrm{Hu}, \mathrm{L} .$, see Acosta-Calderon, C. A.

Ishiguro, H., see Hayashi, K.

Ishiguro, H., see Matsumura, R.

Ishiguro, H., see Shiomi, M.

Ivancevic, T. T., see Ivancevic, V. G.

Ivancevic, V. G. and Ivancevic, T. T., Human versus Humanoid Robot Biodynamics

Ivancevic, V. G. and Sharma, S., Complexity in Human and Humanoid Biomechanics

Jovanović, M., see Vukobratović, M.

Kalkan, S., see Kraft, D.

Kallmann, M., see Carpin, S.

Kanda, T., see Hayashi, K.

Kanda, T., see Nomura, T.

Kanda, T., see Shiomi, M.

Kanniah, J. and Lwin, Z. N., ZMP Compliant Gait Generation Strategies for Seven-Mass Biped Robots

Kato, K., see Nomura, T.

Kawamura, K., Gordon, S. M., Ratanaswasd, P., Erdemir, E. and Hall, J. F., Implementation of Cognitive Control for a Humanoid Robot

Kelley, R., King, C., Tavakkoli, A., Nicolescu, M., Nicolescu, M. and Bebis, G., An Architecture for Understanding Intent Using a Novel Hidden Markov Formulation

Kiener, J., see Friedmann, M.

King, C., see Kelley, R.

Koene, A., see Morén, J.

Koizumi, S., see Shiomi, M.

Kraft, D., Pugeault, N., Başeski, E., Popović, M., Kragić, D., Kalkan, S., Wörgötter, F. and Krüger, N., Birth of the Object: Detection of Objectness and Extraction of Object Shape Through Object-Action Complexes

Kragić, D., see Kraft, D.

Krüger, N., see Kraft, D.

Lacroix, J., Postma, E., van den Herik, J. and Murre, J., Toward a Visual Cognitive System Using Active Top-Down Saccadic Control

Lwin, Z. N., see Kanniah, J.

Mansard, N., see Stasse, O.

Matsumura, R. and Ishiguro, H., Development of a High-Performance Humanoid Soccer Robot
2 (2008) 161

4 (2008) 639

3 (2008) 397

$3(2008) 437$

3 (2008) 397

1 (2008) 67

3 (2008) 353

4 (2008) 587

4 (2008) 699

4 (2008) 699

4 (2008) 679

4 (2008) 639

2 (2008) 247

3 (2008) 481

1 (2008) 67

1 (2008) 25

4 (2008) 587

4 (2008) 609

1 (2008) 25

$4(2008) 547$

2 (2008) 203

3 (2008) 417

2 (2008) 203

1 (2008) 3

4 (2008) 587

2 (2008) 247

2 (2008) 247

2 (2008) 247

2 (2008) 225

4 (2008) 609

2 (2008) 287

3 (2008) 353 
Mayer, N. M. and Asada, M., Robocup Humanoid Challenge

$3(2008) 335$

Mazzanti, F., see Menegatti, E.

3 (2008) 523

McMillen, C., see Veloso, M.

3 (2008) 457

Menegatti, E., Silvestri, G., Pagello, E., Greggio, N., Cisternino, A.,

Mazzanti, F., Sorbello, R. and Chella, A., 3D Models of Humanoid

Soccer Robot in USARSim and Robotics Studio Simulators

Milinović, M., see Vukobratović, M.

Miyashita, T., see Hayashi, K.

Mohan, R. E., see Acosta-Calderon, C. A.

Morén, J., Ude, A., Koene, A. and Cheng, G., Biologically Based

Top-Down Attention Modulation for Humanoid Interactions

Murre, J., see Lacroix, J.

Nicolescu, M., see Kelley, R.

Nomura, T., Suzuki, T., Kanda, T., Han, J., Shin, N., Burke, J. and Kato, K., What People Assume About Humanoid and Animal-Type Robots: Cross-Cultural Analysis Between Japan, Korea, and the United States

$3(2008) 523$

$1(2008) 119$

1 (2008) 67

$3(2008) 397$

$1(2008) 3$

$\mathbf{2}(2008) 225$

$\mathbf{2}(2008) 203$

Omrčen, D., see Ude, A.

Pagello, E., see Carpin, S.

Pagello, E., see Menegatti, E.

Petters, S., see Friedmann, M.

Popović, M., see Vukobratović, M.

Popović, M., see Kraft, D.

Postma, E., see Lacroix, J.

Potkonjak, V., see Vukobratović, M.

Potkonjak, V., see Vukobratović, M.

Pugeault, N., see Kraft, D.

Raković, M., see Vukobratović, M.

Raković, M., see Vukobratović, M.

Ratanaswasd, P., see Kawamura, K.

Roth, M., see Veloso, M.

Saïdi, F., see Stasse, O.

Sakamoto, H., see Friedmann, M.

Sauser, E., see Hersch, M.

Sharma, S., see Ivancevic, V.

Shin, N., see Nomura, T.

Shiomi, M., Kanda, T., Koizumi, S., Ishiguro, H. and Hagita, N., Group Attention Control for Communication Robots

Silvestri, G., see Menegatti, E.

Sorbello, R., see Menegatti, E.

Stasse, O., Saïdi, F., Yokoi, K., Verrelst, B., Vanderborght, B., Davison, A., Mansard, N. and Esteves, C., Integrating Walking and Vision to Increase Humanoid Autonomy

Stryk, O. V., see Friedmann, M.

Stückler, J., see Behnke, S.

Suzuki, T., see Nomura, T.

Tavakkoli, A., see Kelley, R.

Thomas, D., see Friedmann, M.

Tondu, B., A Kinematic Model of the Upper Limb with a Clavicle-Like Link for Humanoid Robots

1 (2008) 25

$\mathbf{2}(2008) 267$

$3(2008) 481$

$3(2008) 523$

$3(2008) 417$

$4(2008) 639$

2 (2008) 247

2 (2008) 225

1 (2008) 119

4 (2008) 639

2 (2008) 247

1 (2008) 119

4 (2008) 639

4 (2008) 547

3 (2008) 457

2 (2008) 287

3 (2008) 417

2 (2008) 161

4 (2008) 679

1 (2008) 25

4 (2008) 587

3 (2008) 523

3 (2008) 523

2 (2008) 287

3 (2008) 417

3 (2008) 375

1 (2008) 25

2 (2008) 203

3 (2008) 417

1 (2008) 87 
Ude, A., Omrčen, D. and Cheng, G., Making Object Learning and Recognition an Active Process

Ude, A., see Morén, J.

Vail, D., see Veloso, M.

van den Herik, J., see Lacroix, J.

Vanderborght, B., see Stasse, O.

Veloso, M., Armstrong-Crews, N., Chernova, S., Crawford, E., McMillen, C., Roth, M., Vail, D. and Zickler, S., A Team of Humanoid Game Commentators

$1(2008) 3$

3 (2008) 457

$2(2008) 225$

2 (2008) 287

Verrelst, B., see Stasse, O.

Vukobratović, M., Borovac, B., Raković, M., Potkonjak, V. and Milinović, M., On Some Aspects of Humanoid Robots Gait Synthesis and Control at Small Disturbances

Vukobratović, M., Herr, H. M., Borovac, B., Raković, M., Popovic, M., Hofmann, A., Jovanović, M. and Potkonjak, V., Biological Principles of Control Selection for a Humanoid Robot's Dynamic Balance Preservation

Wollherr, D., see Bauer, A.

Wörgötter, F., see Kraft, D.

Yokoi, K., see Stasse, O.

Yue, P. K., see Acosta-Calderon, C. A.

Zhang, L. and Zhou, C., Lie Group Formulation for Analysis of Kicking Motion in Humanoid Soccer Robots

Zhou, C., see Acosta-Calderon, C. A.

Zhou, C., see Hu, L.

Zhou, C., see Zhang, L.

Zickler, S., see Veloso, M. 